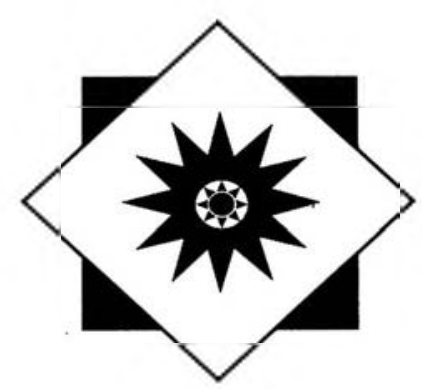

\title{
INNOVATIONS
}

\section{Newspaper indexing with Pro-Cite}

\author{
By Michael D. Cramer
}

\author{
Science Reference Librarian \\ Virginia Polytechnic Institute \& State University
}

\author{
and Mary J. Markland
}

\author{
Science Reference Librarian \\ North Dakota State University
}

In an effort to gain better access and control of our local newspapers the University Libraries at Virginia Tech began an innovative indexing method in 1985 . The $3^{\prime \prime} \times 5^{\prime \prime}$ card file that had been used was time-consuming to maintain, had weak subject access, and was never up-to-date. When the decision was made to automate the newspaper index there were two objectives. The first objective was stronger and easier subject access to our local newspapers; the second objective was an index which could store information compactly as well as economically.

The newspapers indexed are: The Roanoke Times and World News-published seven days a week in our closest large city; the News Messenger-a community newspaper published five times a week; the Collegiate Times - a twice-weekly student publication covering campus events; and the Spectrum-a once-a-week publication covering campus events for faculty and staff.

After examining several software packages the decision was made to utilize Pro-Cite, produced by Personal Bibliographic Software. Pro-Cite is designed to handle large amounts of bibliographic information. One Pro-Cite work form is designed specifically for newspapers. Fields within this work form may vary in length. In addition Pro-Cite allows for truncation of search terms and is capable of Boolean searching. It can be run on IBM personal computers and compatibles which the University Libraries uses. Currently we are running version 1.4, August 1988. The software resides on an IBM OS/2 terminal which is attached to an Epson EX800 printer and an Omega Bernoulli box two-cartridge system.
Because the Pro-Cite software makes possible the creation of various databases (files), we elected to create 10 databases which suit our particular needs. These databases and their names represent those broad areas about which our users, principally undergraduates and non-university individuals, most frequently request information. They are: the VA (for Virginia) file, the RO (for Roanoke) file, the New River (for the New River Valley) file, the MontCo (for Montgomery County) file, the CBurg (for Christianburg) file, the Blksburg (for Blacksburg) file, the Radford file, the VaTech file, the Sports (VaTech only) file, the Univ (Virginia colleges and universities) file. Currently these ten files total 17,809 records.

Our indexing information makes use of $6-8$ lines on a Pro-Cite newspaper workform:

1. Title line (article title)

2. NEWS line (newspaper name)

3. DATE line (newspaper date)

4. LOC line (section and page number of article)

5. INDEX line (contains those keywords underlined within the article)

6. CALLline (newspaper call number)

7. Note line (used occasionally to note the presence of charts, maps, etc. or to indicate that the article was part of a series.

8. Author line (occasionally used to note a specific, important author - for example the Universitypresident).

The indexing process has the following routine. As each issue of a paper is received it is logged on a receipt calendar. The reference librarian respon- 
sible for the project scans the paper for pertinent articles. The file name is written by the title for each selected article and the keywords are underlined. If additional terms are necessary these are written down as well. The number of keywords varies from article to article but we aim for a maximum of 3-6. As a rule each article is assigned to one file. However, at times the nature of an article is such that it may be included in additional files. A student employed 8-10 hours perweek takes the annotated newspapers and inputs the data via Pro-Cite onto a $31 / 2$-inch disk. Once a week these files are merged with the master file on the Bernoulli cartridge. The master file is then copied.

As knowledge of the database has spread, library patrons often approach the main reference desk specifically requesting a newspaper search. Others are identified during the reference interview. In either case a brief search request form is completed and the patron is asked to return in 24 hours for the results. With the database and equipment located two floors above the reference desk we have found the one-day delay necessary. The searcher retains a copy of the search for statistics but also frequently notes search terms and results for possible future reference. When several members of an English class request information on the same topic this last step has been particularly useful. The printout received by the patron contains all the information in the Pro-Cite record. With truncation and Boolean searching possible on Pro-Cite, our suc- cess rate has been affected more by the content of the database than anything else.

Two areas of difficulty have arisen over the past four years: documentation and terminology. Current documentation covers the technical aspects of the database (record format, merging, etc.). We are preparing to write formal guidelines for the indexing process itself such as the selection of keywords, and the inclusion criteria for articles. Our second problem area is also being resolved. Because we rely on keyword access to the index it is important to maintain keyword uniformity. However, the papers have varied their terminology over time and that is reflected in the database. An excellent example of this is an important local highway project. At times this has been referred to as the Route 460 bypass, the Route 460 corridor, and the direct link. To resolve these variations a list of keywords will be produced for each file. Uniformity of terms will be re-established, typographical errors corrected, and a new list of keywords produced. The indexer will be able to refer to the thesaurus while selecting keywords to assure consistency. Terms will be added, deleted, or changed as necessary. Those records having terms which are deleted or changed will be edited.

Our newspaper index utilizing Pro-Cite provides timely access to local papers. By giving a unique twist to Pro-Cite's capacity to handle large amounts of bibliographic information, we have met both of the objectives identified four years ago.

\title{
Students and support staff on the reference desk
}

\author{
By Lynn Westbrook
}

Coordinatorfor Reference and Instruction

Undergraduate Library, University of Michigan

As yet another library school dissolves, the need for quality practicum opportunities for students at the remaining institutions grows. As various groups within ALA wrestle with the thorny problem of recruiting a diverse population of graduate library science students, the need for meaningful jobs during graduate school becomes a significant piece of the problem for many potential students who cannot afford to shelve books while getting a library science degree when they could be doing laboratory work while getting a pharmacy degree.

These situations share a common, if partial, solu- tion which provides major benefits for those libraries able to practice it. By creating and publicizing a well-crafted reference and/or BI training program, libraries provide four contributions to the profession: excellent practical experience for students who can get course credit for library work; the opportunity for working library science students to gain the type of expertise which will give them a real boost in their first job hunt; the opportunity for undergraduates to experience and consider the possibility of library science as a profession; and valuable assistance for their own departments. 